\title{
Recovery of Surgical Workflow Without Explicit Models
}

\author{
Seyed-Ahmad Ahmadi ${ }^{1}$, Tobias Sielhorst ${ }^{1}$, Ralf Stauder ${ }^{1}$, Martin Horn $^{1}$, \\ Hubertus Feussner ${ }^{2}$, and Nassir Navab ${ }^{1}$ \\ ${ }^{1}$ Chair for Computer Aided Medical Procedures (CAMP), TU Munich, Germany \\ ${ }^{2}$ Chirurgische Klinik und Poliklinik, Klinikum Rechts der Isar, TU Munich, Germany
}

\begin{abstract}
Workflow recovery is crucial for designing context-sensitive service systems in future operating rooms. Abstract knowledge about actions which are being performed is particularly valuable in the OR. This knowledge can be used for many applications such as optimizing the workflow, recovering average workflows for guiding and evaluating training surgeons, automatic report generation and ultimately for monitoring in a context aware operating room.

This paper describes a novel way for automatic recovery of the surgical workflow. Our algorithms perform this task without an implicit or explicit model of the surgery. This is achieved by the synchronization of multidimensional state vectors of signals recorded in different operations of the same type. We use an enhanced version of the dynamic time warp algorithm to calculate the temporal registration. The algorithms have been tested on 17 signals of six different surgeries of the same type. The results on this dataset are very promising because the algorithms register the steps in the surgery correctly up to seconds, which is our sampling rate. Our software visualizes the temporal registration by displaying the videos of different surgeries of the same type with varying duration precisely synchronized to each other. The synchronized videos of one surgery are either slowed down or speeded up in order to show the same steps as the ones presented in the videos of the other surgery.
\end{abstract}

\section{Introduction and Related Work}

Surgical workflow recovery is based on the hypothesis that surgeries of one certain type can be compared to each other. In general, these procedures are never performed in exactly the same way, since the experience of the surgeon and the anatomy of the patient vary and complications may occur. Despite these differences, surgeries of one type generally share a common sequence of events, which is typical for the procedure. As Herfarth denotes, a thorough and smooth integration of surgical workflow in the clinical framework is becoming more and more necessary. He postulates that surgical units have to become specialized and dedicated to only one type of surgery up to a specialization on individual organs [1]. The recovery of workflow is one of the crucial tasks towards the long-term goal of opinionated consultancy inside the OR, which has been drafted by experts during discussions in the OR2020 workshop of 2004 [2]. The benefits of a 
system which is aware of and reactive towards the current workflow are for the medical as well as for the administrative side of a hospital.

Riedl et al. postulate in their work more transparency of events within the OR in order to improve efficiency of room and tool usage [3]. Aggarwal et al. 4. have discussed the possibilities of surgery simulation for training purposes, since it has the potential to reduce the occurrence of adverse events. Their OR simulation environment records various data streams, both video and audio, for the assessment of surgical and team skills through expert surgeons in debriefing sessions. Rosen et al. 5 read data from force/torque sensors at laparoscopic instruments and analyse video data about the tool/tissue interaction for the segmentation of movements using hidden markov models (HMMs). Their objective is to evaluate surgical skills. Lin et al. 6] follow a similar approach and propose a system for automated workflow recovery based on motion segmentation with linear discriminant analysis, bayes classifiers and HMMs. Their framework is the analysis of robot-assisted movements with the Intuitive Surgical's da Vinci system during a suturing task. Their aims are the evaluation of surgical skill levels and training of inexperienced surgeons. Strauss et al. [7] have created manual protocols of endoscope usage time, pose changes and instrument changes in order to prove the usability and necessity of an ontology for the description of surgical workflow. They postulate and conceptualize a mechatronic assistance system for endoscope guidance. Sielhorst et al. 8] synchronize 3D hand movements of a physician using Dynamic Time Warping (DTW). They use this in an AR setting for the synchronized playback of movements from trainees and experts for training and evaluation purposes.

While 5] and 6] have been able to segment single and isolated tasks within a whole surgical procedure, we see many benefits of a system that is able to understand the overall workflow of a complete surgery. In this paper, we propose a DTW-based system, which is specialized, but not confined to the classification and labelling of workflow steps within a complete minimally-invasive procedure. Approaches using Hidden Markov Models allow for modelling the workflow as a non-linear procedure, but they need the definition of a limited number of welldefined states. We present a complementary approach that allows for discovery of these states from a large number of signals acquired during surgeries of the same type. This paper describes the implementation of our approach and first results on six minimally invasive surgeries.

\section{Surgical Environment}

As an exemplary surgery for the workflow retrieval we have chosen cholecystectomy. $95 \%$ of the removal of the gall bladder are performed laparoscopically with a very low conversion rate. In spite of its complexity, the frequent performance of the surgery and its stable outcome make it a practical type of surgery for our kind of analysis. In order to relate to the materials and methods that have been used for workflow recovery in the course of this paper, a short verbal description of the workflow follows. 
After anaesthesia and further preparation of the patient, like disinfection of the abdominal region, a small incision is made at the umbilicus or navel of the patient. The abdomen of the patient is inflated with $\mathrm{CO} 2$ in order to create an abdominal cavity. This marks the beginning of the procedure to us. When the inflation is sufficient, the trocars and the laparoscope for minimally invasive surgery are inserted into the abdomen. A metal rod is inserted through one of the trocars, the liver is pulled up and the gallbladder attached beneath is revealed.

Using a grasper and a dissecting device, the surgeon then identifies the positions of the gallbladder's two main vessels, the common bile duct and the bile artery. These vessels are uncovered from adhesions and adjacent tissue. When sufficiently dissected, they are clamped using a clipping device and subsequently cut using a pair of laparoscopic scissors. As a next step, the gallbladder is removed from the liver. In order to separate the gallbladder from the liver, highfrequency current is applied in two modes, cutting and coagulation mode. The metal tips of most instruments serve as electrodes, which are able to dissect the tissue electrically. During the removal of the gallbladder, it is likely that bleedings are caused in the so-called liver bed area, which is a large portion at the lower side of the liver where the gallbladder used to reside. After the gallbladder has been removed completely, these bleedings are stopped using high-frequency current in coagulation mode. In order to retrieve the gallbladder, a retraction sac is inserted and the gallbladder is put into the plastic bag with two or three graspers. One of the trocars is pulled out and the plastic bag is retracted through the umbilical port. After removal, the trocar is inserted again and in a last and final control phase, the situs is once more controlled for bleedings. The remaining vessel stumps are controlled for correct and clean clipping. A drainage tube is inserted in order to lead away the saline irrigation fluid after surgery. All instruments and trocars are retrieved and the four incisions are closed with stitches. The beginning of the abdominal suturing marks the end of laparoscopic activity and thus the end of the workflow that we are considering for this work.

This sequence of events is specific to a standard laparoscopic cholecystectomy. Although variations in the duration of certain phases may occur due to different complications, the general workflow is systematic and thus reproducible.

\section{Algorithms and Methods}

\subsection{Principle of Workflow Recovery}

In minimally-invasive surgeries, the laparoscopic instruments used by the surgeon strongly correlate with the underlying workflow. We account for the ongoing actions during the procedure with a binary model for the instrument usage:

$$
u(t)= \begin{cases}1 & \text { if the instrument is used at time } t \\ 0 & \text { if the instrument is not used at time } t .\end{cases}
$$

A series of multi-dimensional state vectors over time takes into respect that several instruments can be used simultaneously. Currently, we observe the usage of 17 different laparoscopic instruments. 
Our novel way of workflow recovery is based on the following four steps:

1. Synchronization of events. Using the DTW algorithm, we synchronize different surgeries with variable duration in a non-linear manner. Due to the synchronization of signals, the underlying events and workflow steps are synchronized automatically as well.

2. Creation of an average surgery. An average surgery is created. It reveals events that are common to all procedures.

3. Identification of workflow phases. Common events within the average surgery trigger the start and end points of workflow phases.

4. Obtaining workflow phases for the original surgeries. Using the time mapping from each surgery onto the average, workflow phases for each original surgery can be identified at the end of the procedure.

\subsection{Classical DTW}

The DTW algorithm is able to perform the non-linear synchronization that is necessary for the mapping of our surgery data. DTW was most probably originated in the field of speech recognition [9] and was further used in the synchronization of chemical batch processes [10] as well as in many other fields such as motion detection and segmentation [1] [12] or synchronization [8].

In this paper, only the basic principles of the DTW and the extensions which were necessary for this project shall be made clear. For details and further explanations of the algorithm, it is recommended to refer to [9], 10] and [8].

The DTW is a recursive function, which aims at providing correspondencies reducing the distance between two trajectories $A$ and $B$. For each time point $i$ of curve $A$, the distance is calculated to each time point $j$ of curve $B$ and denoted in a 2D DTW matrix field.

The recursive definition of the DTW algorithm 9] and the principles of dynamic programming [13] have the effect that a trench of lowest values occurs while the DTW matrix is filled. Backtracking this trench yields the so-called warp path $h(k)$, where $k$ denotes a newly established, common timeline of the two curves. This warp path is the optimal linkage between the two timelines $i$ and $j$, so that the curves $A$ and $B$ are mapped onto each other according to their similarities as best as possible.

By using a multi-dimensional distance function $d(i, j)$, we are able to compare the state vectors of our surgeries and obtain the desired optimal DTW time mapping (cf. Figure 1). For our experiments, we made use of the standard Euclidian distance.

\subsection{Creating an Average Surgery}

The creation of an average surgery is crucial for our proposed method of workflow recovery. In order to create such an average, more than two surgeries need to be synchronized onto a common timeline. Although the classic DTW algorithm only allows synchronizing two curves at a time, Wang and Gasser have overcome this 

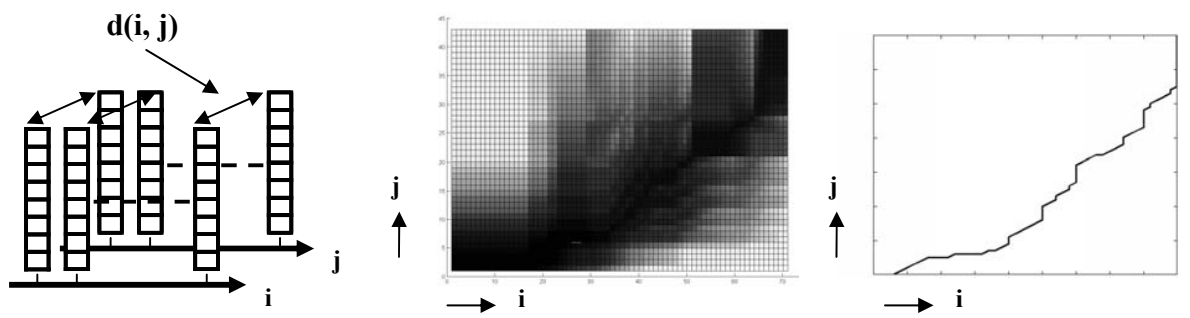

Fig. 1. DTW warp paths synchronize the time axes $i$ and $j$ of two surgeries so that their state vectors are optimally aligned.

problem by making use of the transitive property of the DTW [14] 15]. Using their method, we synchronize an arbitrary number of surgeries by applying the DTW several times according to the following scheme:

- Out of a set of $\mathrm{m}$ surgeries $O P_{i},(i=1 \ldots m)$, one surgery is chosen as a reference curve $O P_{\text {Ref }}$ and all other surgeries are mapped onto the reference using the multi-dimensional DTW. Thus, we obtain $(m-1)$ warp paths $h_{i}(t)$. $h_{i}(t)=D T W\left(O P_{i}, O P_{R e f}\right)$

- The warp paths are interpolated and averaged, yielding a warp path $\mathrm{h}(\mathrm{t})$ between the reference curve and a common, average timing. $h(t)=\frac{1}{m} \sum_{i=1}^{m} h_{i}(t)$

- In the third step, we calculate the desired warp paths $u_{i}(t)$ between the average timing and the timing of each original surgery. These so-called shift functions are calculated by taking the inverse of $h(t)$ as the argument of the warp paths $h_{i}(t): u_{i}(t) \equiv h_{i}\left(h^{-1}(t)\right)$

- Using these shift functions, the instrument state vectors of each surgery can be arranged onto the new, average timeline. Subsequently, the instrument vectors themselves are averaged for each timestamp, yielding the so-called structural average $O P_{A v g}: O P_{A v g}(t)=\frac{1}{m} \sum_{i=1}^{m}\left(u_{i}(t)\right)$

For the reference curve, we chose the one with a duration that is closest to the average duration of the five surgeries. Due to the averaging process, state vectors in $O P_{A v g}$ feature non-binary values as opposed to our original model. These values can be interpreted as quasi-probabilities. Let us assume that at $t_{a}$, $n$ out of the $m$ surgeries from the averaging set had a certain instrument in use. Then, the value for this instrument in the average will be: $O P_{A v g}\left(t_{a}\right)=\frac{n}{m}$

These values are not a probability in the axiomatic sense, but they give an idea of how well an instrument could be synchronized by the algorithm. Furthermore, they reflect how well the surgeries correspond to each other at $t_{a}$. More importantly, these values reveal events, which are typical to the surgical procedure.

\subsection{Weighting of Signals From the Curves}

It makes sense to bias the DTW synchronization towards instruments which have a high relevance for the underlying workflow. For example, the clipping device 

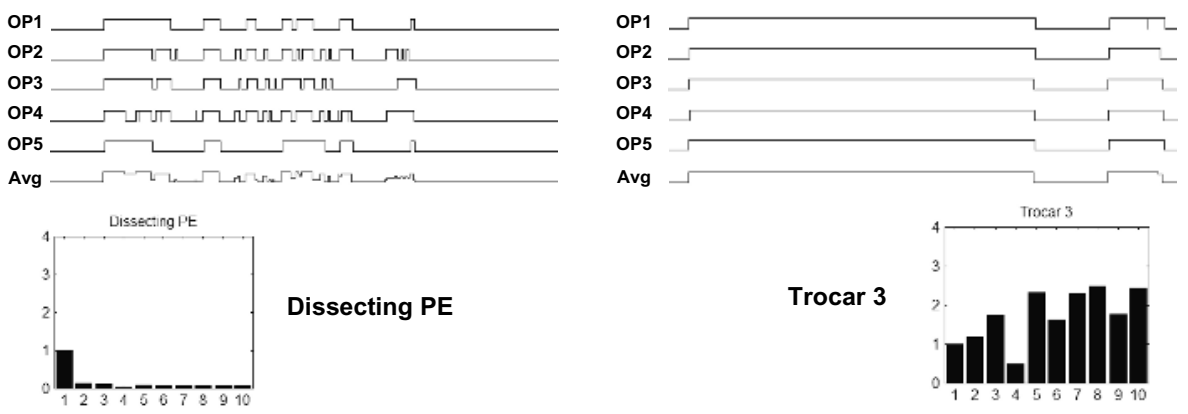

Fig. 2. Weighting of instruments according to their workflow relevance: the better the synchronization, the higher the weight.

clearly indicates the work step of clamping a vessel, while the dissecting device is used as an all-round instrument in many different phases of the surgery.

Mathematically, the bias is achieved by assigning different weights to the instruments during the calculation of the distance function. The problem of weighting certain variables over others in a multi-dimensional setting has been encountered before by the group around Kassidas et al. [10].

Adopting their method, we perform iterative calculations of an average surgery. After every round, each instrument from the state vector is compared to its synchronized equivalent. A new weight is then assigned and subsequently normalized.

$W(j, j)=\left[\sum_{i=1}^{n} \sum_{k=0}^{t_{\text {avg }}}\left[O P_{i}(k, j)-O P_{\text {Avg }}(k, j)\right]^{2}\right]^{-1}, W\left\{n_{\text {Instr }} /\left[\sum_{j=1}^{n_{\text {Instr }}} W(j, j)\right]\right\}$

In simple words, the rule is to give higher weight to those instruments, which could be synchronized very well and lower weight to instruments with only poor synchronization (cf. Figure 2).

\section{Experiments and Results}

\subsection{Workflow Retrieval}

For the evaluation of our developed algorithms, six surgeries were recorded by courtesy of our clinical partner. The average surgery was calculated with five surgeries from the set and after ten iterations of weight updating. The sampling frequency for the instrument state vectors was $1 \mathrm{~Hz}$. The state vectors were manually read out from the laparoscopic video. The sixth surgery was then synchronized to the average in order to evaluate the quality of workflow retrieval for surgeries, which are unknown to the system. The resulting average surgery $O P_{A v g}$ can be seen in Figure 3 .

The curve segment from $O P_{A v g}$ marked with a dashed ellipse demonstrates that common events are carved out while uncommon events tend to be attenuated but not neglected within the average surgery. This happens only due to 


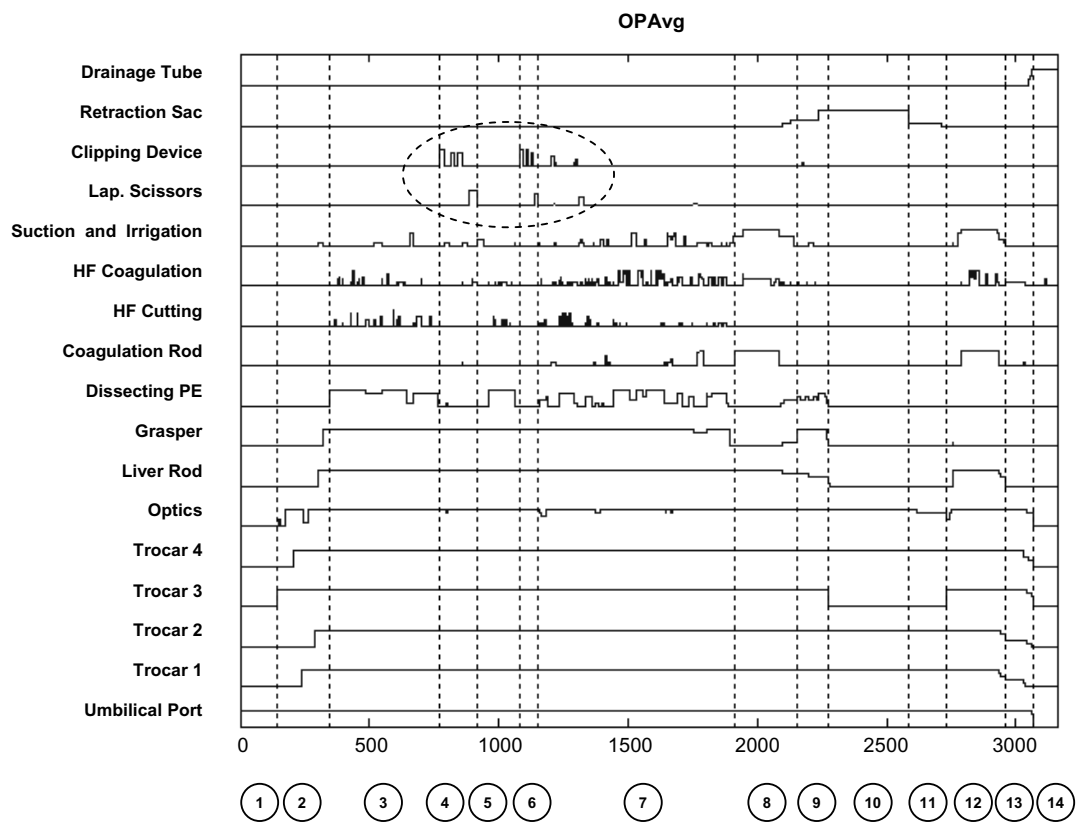

Fig. 3. Average surgery with 14 workflow phases

the synchronization and without prior knowledge or models on the workflow. A short explanation shall substantiate this assertion. For every patient, two main gallbladder vessels have to be severed. This is represented by three high peaks for the clipping device and one high peak for the scissors. For some patients, these vessels have branched, which requires additional clipping and cutting. Since these additional events are rather uncommon, the following spikes have lower amplitudes or lower quasi-probabilities.

State changes from zero to one or one to zero within $O P_{A v g}$ were defined as trigger events for workflow phases. Using these triggers, 14 workflow phases could be identified within the average curve. The segmentation of these phases in the multi-dimensional curves can be seen in Figure 3 , The same trigger events were identified within each original surgery. Thus, two trigger time points exist for each phase in an original surgery. The first is the original time stamp $t$ of the phase beginning. The second one, $t^{*}$, is obtained through the average surgery $O P_{A v g}$ and the shift function $u_{i}(t)$, which connects the timeline of $O P_{i}$ with the one of $O P_{A v g}$.

A deviation of up to five seconds between these timestamps was tolerated. Deviations higher than five seconds were classified as a wrong phase detection. In six procedures with 13 trigger events each, the proposed system was able to identify $92 \%$ of the events correctly. Moreover, $83 \%$ of the correctly classified phases were detected with a precision of one second or less. These results demonstrate that our approach is reliable and promising. The video presentation shows the user interface and some exemplary results. 


\section{Conclusion}

Workflow recovery is a key competency of context-sensitive systems and workflowaware operating rooms. We have shown that our approach of workflow recovery is able to identify common phases in different surgeries of the same type even under the conditions of a real surgery. For this task, an average surgery is computed from a set of signals from exemplary surgeries, which already provides the desired key information for many workflow specific applications. The average curve is achieved by synchronizing multiple procedures and it reveals key events of the procedure. The change of workflow phases can be identified with a reliability of $92 \%$ with a tolerance of 5 seconds. Each of the signals including the video streams from the laparoscope and the video stream of our three external cameras can be shown simultaneously for two or more surgeries. The fine-grained synchronization of the algorithm allows for stretching the playback speed in a way that the surgeries are visualized according the workflow phases. Therefore, each single frame of all 24 videos is appropriately labelled with synchronized workflow information for further studies. The playback is useful for thorough and unprecedented analysis of surgical workflow, educational and training purposes and evaluation of surgical skills.

\section{References}

1. Herfarth, C.: 'lean' surgery through changes in surgical workflow. British Journal of Surgery 90 (2003) 513-514

2. Cleary, K., Chung, H.Y., Mun, S.K.: Or 2020: The operating room of the future. Laparoendoscopic and Advanced Surgical Techniques 15 (2005) 495-500

3. Riedl, S.: Modern operating room management in the workflow of surgery. spectrum of tasks and challenges of the future. Der Chirurg 73 (2002) 105-110

4. Aggarwal, R., Undre, S., Moorthy, K., Vincent, C., Darzi, A.: The simulated operating theatre: comprehensive training for surgical teams. Qual Saf Health Care 13 (2004) 27-32

5. Rosen, J., Solazzo, M., Hannaford, B., Sinanan, M.: Task decomposition of laparoscopic surgery for objective evaluation of surgical residents' learning curve using hidden markov model. Comput Aided Surg. 7 (2002) 49-61

6. Lin, H.C., Shafran, I., Murphy, T.E., Okamura, A.M., Yuh, D.D., Hager, G.D.: Automatic detection and segmentation of robot-assisted surgical motions. In: MICCAI 2005. Volume 3749., Springer (2005) 802-810

7. Strauss, G., Fischer, M., et al., J.M.: Workflow analysis to assess the efficiency of intraoperative technology using the example of functional endoscopic sinus surgery. HNO (2005)

8. Sielhorst, T., Blum, T., Navab, N.: Synchronizing 3d movements for quantitative comparison and simultaneous visualization of actions. In: Proc. IEEE and ACM International on Mixed and Augmented Reality (ISMAR). (2005)

9. Sakoe, H., Chiba, S.: Dynamic programming algorithm optimization for spoken word recognition. IEEE Trans. Acoust. Speech Signal Process. 26 (1978) 43-49

10. Kassidas, A., MacGregor, J.F., Taylor, P.A.: Synchronization of batch trajectories using dynamic time warping. AIChE Journal 44 (1998) 864-875

11. Li, H., Greenspan, M.: Multi-scale gesture recognition from time-varying contours. Tenth IEEE International Conference on Computer Vision (ICCV) 1 (2005) 236-243 
12. Bobick, A.F., Wilson, A.D.: A state-based approach to the representation and recognition of gesture. IEEE Transactions on Pattern Analysis and Machine Intelligence 19 (2005) 1325-1337

13. Bellman, R.E., Dreyfus, S.E.: Applied Dynamic Programming. Princeton University Press (1962)

14. Wang, K., Gasser, T.: Alignment of curves by dynamic time warping. Annals of Statistics 25 (1997) 1251-1276

15. Gasser, T., Wang, K.: Synchronizing sample curves nonparametrically. Annals of Statistics 27 (1999) 439-460 